\title{
GESTIÓN DE RIESGO DE DESASTRES Y RESPONSABILIDAD SOCIAL EN EL GOBIERNO REGIONAL DE TACNA, 2019 ${ }^{1}$
}

\section{DISASTER RISK MANAGEMENT AND SOCIAL RESPONSIBILITY IN THE REGIONAL GOVERNMENT OF TACNA, 2019}

D0I: https://doi.org/10.47796/ra.2021i20.550

JUAN ERIK CABRERA HUAYHUA ${ }^{2}$

Universidad Nacional Jorge Basadre Grohmann, Tacna - Perú

https://orcid.org/0000-0001-6368-7530

juan.cabrera@unjbg.edu.pe

\section{RESUMEN}

El objetivo de esta investigación fue determinar la relación entre la gestión de riesgo de desastres y la responsabilidad social en el Gobierno Regional de Tacna (2019). Se empleó un enfoque cuantitativo tipo básico, nivel correlacional, de diseño no experimental y corte transversal. La muestra estuvo constituida por 174 trabajadores, seleccionados por muestreo aleatorio, quienes respondieron dos instrumentos que miden gestión de riesgo de desastres y responsabilidad social, ambos con validez y confiabilidad. Se reportó que el $64.9 \%$ del personal consideró de nivel regular la gestión de riesgo de desastres característica de esta institución, y que la dimensión más destacada es la gestión correctiva; asimismo, el $67.2 \%$ consideró como nivel regular la responsabilidad social, en el que sobresale el tipo de dimensión externa. Asimismo, se reportó correlación significativa y directa entre ambas variables ( $p$-valor $=0.000$; Rho $=0.793$ ) Se concluye que existe relación significativa directa y alta entre la gestión de riesgo de desastres y la responsabilidad social en el Gobierno Regional de Tacna.

\section{ABSTRACT}

The objective of this research was to determine the relationship between disaster risk management and social responsibility in the Regional Government of Tacna, 2019, a quantitative approach, basic type, correlational level, non-experimental design and cross section was used. The sample consisted of 174 workers, selected by random sampling who answered two instruments that measure disaster risk management and social responsibility, both with validity and reliability. It was reported that $64.9 \%$ of the staff considered the disaster risk management characteristic of this institution to be of a regular level, the most prominent dimension being corrective management; Likewise, $67.2 \%$ considered social responsibility as a regular level, the type of external dimension being the most prominent. Likewise, a significant and direct correlation was reported between both variables ( $p$-value = 0.000 ; Rho $=0.793)$. It is concluded that there is a direct and high significant relationship between disaster risk management and social responsibility in the Regional Government of Tacna.

\footnotetext{
Los resultados de la presente investigación son producto del trabajo de tesis de maestría en Gestión Ambiental y Desarrollo Sostenible de la Escuela de Posgrado de la Universidad Nacional Jorge Basadre Grohmann de Tacna-Perú.

Arquitecto con estudios de posgrado en Gestión Ambiental y Desarrollo Sostenible por la Universidad Nacional Jorge Basadre Grohmann de Tacna - Perú; especialidad en Administración y Gestión Pública; especialista en el Sistema Nacional de Programación Multianual de Inversiones; Gestor de Proyectos con más de 10 años de experiencia en planificación, monitoreo y programación de inversiones.
} 
PALABRAS CLAVE: gestión de riesgo de desastres, responsabilidad social

\section{INTRODUCCIÓN}

Los desastres pueden verse como grandes problemas sociales que afectan a una comunidad, especialmente a aquellos más vulnerables. Aunque muchos fenómenos presentan una naturaleza cíclica, existen otros que tienen una aparición repentina y son los que generan mayor impacto (Oficina para la Coordinación de Asuntos Humanitarios de las Naciones Unidas [OCHA], 2020), como en América Latina que es el continente más afectado por este tipo (Centro de la Investigación sobre la Epidemiología de los Desastres [CRED], 2017). Según el Instituto Geofísico del Perú (IGP, 2020), este país, por su condición geográfica, es susceptible para que ocurran eventos naturales potencialmente muy peligrosos en todo su territorio. Generalmente, las autoridades prestan ayuda a los damnificados luego de ocurrido el siniestro, a través de medidas improvisadas y con bajos niveles de coordinación, de modo que es posible observar situaciones de competencia entre las instancias de gobierno o que la ayuda internacional no es correctamente direccionada; por tanto, se genera un escenario difícil para la rehabilitación y reconstrucción de la población que sigue en expansión y a la vez continúa expuesta al riesgo.

Se entiende gestión de riesgos como el accionar que pretende revertir los elementos de vulnerabilidad que caracterizan a una población: infraestructura, actividades productivas y el ambiente (Comisión Nacional de Prevención de Riesgos y Atención de Emergencias, 2014). Asimismo, Strand et al. (2003) lo definen como un conjunto de
KEYWORDS: disaster risk management, social responsibility.

disposiciones institucionales, administrativas y operacionales que buscan disminuir el impacto de los eventos naturales, ambientales y tecnológicos en la población a través de la creación de medios seguros. En esta misma línea, Lavell (2007) lo define como un conjunto de procesos que permite la formulación e implementación de políticas y estrategias calculadas para reducir y controlar las fragilidades en determinados territorios afectados por el siniestro.

En el territorio peruano, se promulgó la ley No. 29664, que permite la creación del Sistema Nacional de Gestión del Riesgo de Desastres (SINAGERD, 2011), para reemplazar el Sistema Nacional de Defensa Civil (SINADECI) con la finalidad de implementar un nuevo estilo sobre la forma de entender y enfrentar los desastres. Asimismo, se ha observado que existe una explotación descontrolada de los recursos naturales, una posesión irracional de los territorios con fines de vivienda, el incremento de actividades de extracción de tipo agroindustrial y otros; de ahí que el actual objetivo del SINAGERD es impulsar una cultura de prevención más que de reacción, hasta convertirse en una parte esencial de los procesos que desarrollan las entidades públicas: planificación, orden del territorio, inversión, gestión del ambiente y otros. Esta ley No. 29664 describe los componentes de la gestión de riesgo de desastres, los mismos que fueron utilizados como dimensiones en la presente investigación. Estos son gestión prospectiva, definida como el conjunto de acciones que se realizan para evitar un riesgo futuro; gestión correctiva que especifica al conjunto de acciones planificadas con el fin de corregir el 
riesgo existente; gestión reactiva que precisa al conjunto de acciones y medidas destinadas a enfrentar los desastres, sea por un peligro inminente o por materialización del riesgo. Las dos primeras gestiones están a cargo del Centro Nacional de Estimación, Prevención y Reducción del Riesgo de Desastres (CENEPRED) y la tercera a cargo del Instituto Nacional de Defensa Civil (INDECI). Cada uno de ellos comprende indicadores tales como plan de desarrollo, plan de ordenamiento territorial, (gestión prospectiva); acciones de reubicación, acciones de reforzamiento (gestión correctiva), y capacidad de respuesta, preparación para la respuesta y almacenamiento (gestión reactiva).

La ley No. 29664 establece que tanto el gobierno regional como el local deben componer un grupo de coordinación y articulación, el cual deberá denominarse Grupo de Trabajo para la Gestión del Riesgo de Desastres (GTGRD). Este debe tener como objetivo cumplir lo reglamentado en dicha ley en lo que compete a la gestión de riesgo de desastres, y se caracteriza por su articulación interna que permite la implementación y evaluación de los procesos de gestión de riesgo de desastres. Los miembros del GTGRD deben ser autoridades y funcionarios con capacidad de decisión en materias de este tipo para que coordinen las tres áreas de gestión (prospectiva, correctiva y reactiva) y las incorporen al momento de la planificación, ordenamiento territorial, análisis de vulnerabilidad y nivel de riesgo.

Acerca de la responsabilidad social, Rodríguez (2006) la definió como un modelo organizacional caracterizado por la combinación de eficiencia y equidad con el fin de buscar crear riqueza de largo plazo de manera sostenible, en otras palabras, que priorice la preservación del capital medioambiental asociado al desarrollo del capital físico y financiero de la entidad. Sin embargo, para Castillo (2005) la responsabilidad social de una entidad se logra desarrollar con obligación moral y ética respecto al desarrollo de sus habitantes que viven en la zona de influencia de sus actividades. Benavides y Gastelumendi (2010) al tratar de explicitarla han resaltado dos áreas: interna y externa, las mismas que son consideradas en la presente investigación. Por un lado, la responsabilidad social interna se define por la relación entre la entidad con cada uno de los trabajadores y sus familias, y abarca aspectos como la capacitación técnica, la superación personal y las relaciones laborales, además de la comunicación frecuente de los directivos con el personal a cargo, la existencia de un código de ética, el priorizar la salud y seguridad laboral, entre otros. Por otro lado, la responsabilidad social externa implica la relación de la entidad con su medio ambiente, la comunidad y la sociedad en general; en otras palabras, la relación con los grupos de interés en lo que respecta a las actividades priorizadas relacionadas con la salud, educación, cultura, medio ambiente, deportes y otros. A partir de ello, se puede establecer los indicadores para cada área. Así la responsabilidad social interna comprende el impulso al desarrollo económico y social, prácticas responsables con los grupos de interés, respeto al medio ambiente, relación con la sociedad y el Estado; mientras que la responsabilidad social externa abarca indicadores como la gestión de los recursos humanos, salud y seguridad en el trabajo, adaptación al cambio.

Alayo (2018) afirmó que un gran porcentaje del riesgo análogo a los fenómenos naturales se originó en características perceptuales, debido a que los seres humanos piensan que los fenómenos de evolución rápida (por ejemplo, los movimientos sísmicos) ocurren rara vez $y$, por tanto, no le dan la atención debida proporcional según el nivel de impacto y continúan con la construcción de sus viviendas en zonas de riesgo, pese a la identificación y notificación de las mismas por la autoridad competente. Estos procedimientos cuestionan el compromiso del ser humano frente a la actuación entre naturaleza y sociedad. 
Resulta clave que las autoridades y funcionarios, responsables de implementar la política de gestión de riesgo de desastres en Tacna, tengan claridad acerca de la percepción de estos riesgos del personal que labora en el Gobierno Regional, tanto en su labor prospectiva, reactiva como correctiva, puesto que ello permitirá identificar características de mejora para implementar un trabajo más organizado, que reduzca los riesgos a los que está expuesta la población regional. Así también, facilitará contar con información clara sobre el nivel de responsabilidad social respecto al fortalecimiento de las capacidades del personal de la entidad con el objetivo de implementar adecuadamente las políticas públicas regionales, el impulso al desarrollo económico y gestión del medio ambiente.

Es por ello que la presente investigación tuvo como objetivo determinar la relación entre la gestión de riesgo de desastres y la responsabilidad social en el Gobierno Regional de Tacna durante el 2019.

\section{MATERIALES Y MÉTODOS}

La investigación fue de tipo pura o básica, de nivel correlacional y utilizó un diseño no experimental y corte transversal (HernándezSampieri y Mendoza, 2018).

La población de estudio estuvo constituida por 317 trabajadores que laboran en la sede principal del Gobierno Regional de Tacna. Se utilizó un tipo de muestreo aleatorio y se seleccionó solo a 174 miembros.

Se empleó la técnica de la encuesta y se aplicaron dos instrumentos. El primero fue un cuestionario sobre la gestión de riesgo de desastres que percibe el personal del Gobierno Regional de Tacna. Estuvo constituido por 16 ítems agrupados en tres dimensiones: gestión prospectiva (ítems 01,02,03,04, 05, 06, 07), gestión correctiva (ítems $08,09,10,11,12$ ) y gestión reactiva (ítems $13,14,15,16)$. El cuestionario utilizó la escala Likert de 5, los resultados se categorizaron en bajo, regular y alto, según dimensiones y el total. Acerca de la validación, este se realizó a través del criterio de juicio de expertos y se consultó a tres de ellos, quienes emitieron un dictamen calificando al instrumento como excelente, con un nivel de acuerdo del $95 \%$. Asimismo, con respecto a la confiabilidad, esta se midió a través de una prueba piloto a 40 trabajadores y se obtuvo un Alpha de Cronbach de 0.964 .

El segundo instrumento fue un cuestionario sobre el nivel de responsabilidad social que caracteriza al Gobierno Regional de Tacna. Estuvo constituido por 20 ítems agrupados bajo dos dimensiones: interna (ítems del 01 al 10) y externa (ítems del 11 al 20), y utilizó la escala Likert de 5 grados. Los resultados se categorizaron en bajo, regular y alto, según dimensiones y el total. La validación se realizó a través del criterio de juicio de expertos y se consultó a tres de ellos, quienes calificaron de excelente al instrumento, con un nivel de acuerdo del 95\%. La confiabilidad se evaluó a través de una prueba piloto con 40 trabajadores y se obtuvo un Alpha de Cronbach de 0.968 , con lo cual se halló que el instrumento presenta buenos índices de confiabilidad (George y Mallery, 2003) para ser utilizado en el trabajo de campo.

Para el análisis de datos se aplicó el software SPSS versión 24.0 y se utilizó el coeficiente de correlación Rho de Spearman, cuyo índice oscila desde -1 a +1 y la interpretación corresponde a baja $(0.00$ a $\pm 0.39)$, moderada ( \pm 0.40 a \pm 0.59$)$, alta ( \pm 0.60 a $\pm 0.79)$ y muy alta $( \pm 0.80 \mathrm{a} \pm 1.00)$.

\section{DESARROLLO}

Se trabajó con cada instrumento en una muestra piloto de 40 personas lo que permitió 
obtener los puntajes de confiabilidad. Además, se enviaron los cuestionarios a tres jueces expertos a partir de lo cual se obtuvo las puntuaciones de validez. Posteriormente, se realizó la toma de datos a las personas del Gobierno Regional de Tacna. Cabe resaltar que el tipo de fuente de información usada fue primaria, es decir, los datos fueron proporcionados por el propio personal que labora en la sede principal del Gobierno Regional de Tacna.

\section{RESULTADOS}

En la Tabla 1 se observa que el $66.1 \%$ del personal evaluado en la sede principal del Gobierno Regional de Tacna califica la gestión prospectiva de regular y un $29.9 \%$ como alta, lo que indica que los trabajadores consideran que el nivel de planificación de acciones para evitar riesgos futuros no es óptimo. Según el análisis por pregunta, el personal considera que la entidad debería desarrollar espacios de debate regional sobre lo avanzado en este tipo de gestión y que se debería priorizar la utilización del enfoque de gestión de riesgos para que sean posibles los proyectos de inversión. Sobre la gestión correctiva, el 59.8\% la considera como regular y un $34.5 \%$, de nivel alto, lo que refleja que los trabajadores consideran el nivel de planificación de acciones para corregir el riesgo como un nivel aceptable. Según el análisis por ítems, se destaca que el Gobierno Regional de Tacna cuenta con lineamientos para la rehabilitación, planes de emergencia y evaluación de daños, aunque también los encuestados señalan que la entidad debería contar con personal especializado que ejecute la política de gestión de riesgo de desastres, asimismo, que debería existir mayor transparencia en los mecanismos de financiamiento público.

En cuanto a la gestión reactiva, un $74.1 \%$ de los encuestados la considera de nivel regular y un $24.7 \%$, alta. A partir de ello, se interpreta que el personal considera no óptimo el nivel de precisión sobre las acciones y medidas efectuadas para enfrentar los desastres. Según el análisis por preguntas, se destaca que el Gobierno Regional de Tacna promueve simulacros naturales, sin embargo, dicha entidad debería tener mayor claridad al momento de implementar los procesos de rehabilitación y reconstrucción luego del desastre $y$, además, promover reuniones frecuentes de trabajo para decidir las medidas correctivas. Finalmente, en la Tabla 2 se observa la categorización de la variable Gestión de Riesgo de Desastres, en la que un $64.94 \%$ la calificó como regular y un 33.33\%, como alta, lo cual indica que la percepción del personal sobre esta variable es de nivel aceptable.

Tabla 1. Resultados por Dimensiones Acerca de la Gestión de Riesgo de Desastres

\begin{tabular}{c|c|c|c|c|c|c}
\hline \multirow{2}{*}{ Dimensiones } & \multicolumn{2}{|c|}{ Gestión prospectiva } & \multicolumn{2}{c|}{ Gestión correctiva } & \multicolumn{2}{c}{ Gestión reactiva } \\
\cline { 2 - 7 } & Frecuencia & Porcentaje & Frecuencia & Porcentaje & Frecuencia & Porcentaje \\
\hline Bajo & 7 & $4.0 \%$ & 10 & $5.7 \%$ & 2 & $1.1 \%$ \\
Regular & 115 & $66.1 \%$ & 104 & $59.8 \%$ & 129 & $74.1 \%$ \\
Alto & 52 & $29.9 \%$ & 60 & $34.5 \%$ & 43 & $24.7 \%$ \\
Total & 174 & $100.0 \%$ & 174 & $100.0 \%$ & 174 & $100.0 \%$ \\
\hline
\end{tabular}

Fuente: Elaboración propia 
Tabla 2. Resultados por Categorías de

la Variable Gestión de Riesgo de Desastres

\begin{tabular}{c|c|c}
\hline $\begin{array}{c}\text { Gestión de riesgo } \\
\text { de desastres }\end{array}$ & Frecuencia & Porcentaje \\
\hline Bajo & 3 & 1.7 \\
Regular & 113 & 64.9 \\
Alto & 58 & 33.3 \\
Total & 174 & 100.0 \\
\hline
\end{tabular}

Fuente: Elaboración propia

Tabla 3. Resultados por Dimensiones Acerca de la Responsabilidad Social

\begin{tabular}{c|c|c|c|c}
\hline \multirow{2}{*}{ Dimensiones } & \multicolumn{2}{|c|}{ Interna } & \multicolumn{2}{c}{ Externa } \\
\cline { 2 - 5 } & Frecuencia & Porcentaje & Frecuencia & Porcentaje \\
\hline Bajo & 3 & 1.7 & 3 & 1.7 \\
Regular & 113 & 64.9 & 113 & 64.9 \\
Alto & 58 & 33.3 & 58 & 33.3 \\
Total & 174 & 100.0 & 174 & 100.0 \\
\hline
\end{tabular}

Fuente: Elaboración propia

En la Tabla 3, se muestra los resultados con respecto a la variable Responsabilidad Social en su dimensión interna: el $60.9 \%$ del personal de la sede principal del Gobierno Regional de Tacna considera que dicha responsabilidad es de nivel regular, y el $19.5 \%$, de nivel alto, lo cual refleja la percepción del personal respecto de la relación no óptima entre las autoridades de esta institución con cada trabajador y sus familias. Asimismo, según el análisis por ítems, lo que destaca es que el personal proporciona las condiciones básicas para cumplir con eficiencia la labor encargada, sin embargo, se deberían incentivar actividades que desarrollen capacidades sinérgicas entre las áreas de trabajo. Con respecto a la dimensión externa: el 54.6\% refirió el despliegue de esta acción como regular; el 43.7\%, como alta. Esto refleja que la relación de la entidad con el medio ambiente es aceptable. En el análisis por ítems, se subraya que la entidad prioriza dar un trato cordial y mantener empatía con la población, aunque lo que se requiere mejorar es la prioridad para hacer frente a los problemas ambientales y la existencia de una gestión de puertas abiertas en relación a la población.

Finalmente, la Tabla 4 expone los resultados de la variable Responsabilidad Social, en la que un $67.2 \%$ de los trabajadores del Gobierno Regional de Tacna considera que aquella es de nivel regular, y un $30.5 \%$, de nivel alto, lo cual indica que el nivel de responsabilidad social es aceptable.

Tabla 4. Resultados por Categorías de la Variable Responsabilidad social

\begin{tabular}{c|c|c}
\hline $\begin{array}{c}\text { Responsabilidad } \\
\text { social }\end{array}$ & Frecuencia & Porcentaje \\
\hline Bajo & 4 & 2.3 \\
Regular & 117 & 67.2 \\
Alto & 53 & 30.5 \\
Total & 174 & 100.0 \\
\hline
\end{tabular}

Fuente: Elaboración propia

Tabla 5. Índice de Correlación entre la Responsabilidad Social y la Gestión de Riesgo de Desastres

\begin{tabular}{|c|c|c|}
\hline \multicolumn{2}{|r|}{ Coeficiente de correlación } & Responsabilidad social \\
\hline \multirow{4}{*}{ 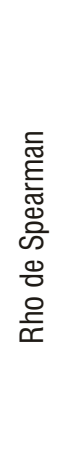 } & $\begin{array}{l}\text { Gestión de riesgo de desastres } \\
\text { Sig. (bilateral) } \\
n\end{array}$ & $\begin{array}{l}0.793^{* \star} \\
0.000 \\
174\end{array}$ \\
\hline & $\begin{array}{l}\text { Dim.1 Gestión prospectiva } \\
\text { Sig. (bilateral) } \\
\mathrm{n}\end{array}$ & $\begin{array}{l}0.728 * \star \\
0.000 \\
174\end{array}$ \\
\hline & $\begin{array}{l}\text { Dim.2 Gestión correctiva } \\
\text { Sig. (bilateral) } \\
n\end{array}$ & $\begin{array}{l}0.782^{* *} \\
0.000 \\
174\end{array}$ \\
\hline & $\begin{array}{l}\text { Dim.3 Gestión reactiva } \\
\text { Sig. (bilateral) } \\
n\end{array}$ & $\begin{array}{l}0.778^{* *} \\
0.000 \\
174\end{array}$ \\
\hline
\end{tabular}

**La correlación es significativa en el nivel 0.01 (bilateral). Fuente: Elaboración propia

El análisis de correlación entre la responsabilidad social y la gestión de riesgo de desastres obtuvo un $p$-valor $=0.000<0.05$. De ahí se concluye que existe relación significativa y positiva entre ambas variables; asimismo, el valor de Rho $=0.793$ se interpreta como una correlación alta. De igual manera, se verifica una correlación significativa y alta en las tres 
dimensiones de la gestión de desastres y la responsabilidad social: gestión prospectiva ( $p$ valor $=0.00 ;$ Rho $=0.728)$; gestión correctiva $(p-$ valor $=0.00$; Rho $=0.782)$; gestión reactiva $(p-$ valor $=0.00$; Rho $=0.778$ ).

\section{DISCUSIÓN}

El objetivo de la presente investigación fue determinar la relación entre la gestión de riesgo de desastres y la responsabilidad social en el Gobierno Regional de Tacna (2019). Se halló que sí existe relación significativa entre ambas variables, lo cual indica que la forma de gestionar los desastres tanto en el ámbito prospectivo, correctivo y reactivo dentro de la institución permite el fortalecimiento de la política de responsabilidad social interna y externa. Esto se daría según el trato digno al personal y una implementación de acciones específicas en temas ambientales, económicos y sociales de la región. Estos resultados concuerdan con lo expuesto por Quispe (2017) quién encontró una relación directa y significativa entre la responsabilidad social y gestión de riesgos en la Municipalidad Provincial de Ica ( $\rho$-valor $=0.000$; Rho $=0.774)$; asimismo con Montañez (2017) quien afirma que existe relación directa y alta entre ambas variables en la Municipalidad Provincial de Yungay (valor de $r$ $=0.722$ ). También el estudio de Bobadilla y Sandoval (2015) reporta que existe influencia de la responsabilidad social en la imagen institucional en la empresa de transporte Rodrigo Carranza S.A.C. en Trujillo. Finalmente, Ochante (2018) concluye que existe relación positiva y alta entre la ejecución de obras públicas y la responsabilidad social ( $\mathrm{Rho}=0.709$ y valor de $p=0.000$ ) en la Municipalidad Provincial de Ica.

\section{CONCLUSIONES}

Se concluye que existe relación significativa y alta entre las variables gestión prospectiva de riesgo de desastres y responsabilidad social; gestión correctiva de riesgo de desastres y responsabilidad social; gestión reactiva de riesgo de desastres y responsabilidad social. Finalmente, a partir de ello se puede aseverar que existe relación significativa y alta entre la gestión de riesgo de desastres y la responsabilidad social en el Gobierno Regional de Tacna (2019).

\section{REFERENCIAS BIBLIOGRÁFICAS}

Alayo, L. (2018). Los desastres y el riesgo. https://www.perusinriesgodedesastres.com/ar t\%C3\%ADculos/

Benavides, M. y Gastelumendi, G. (2010). Responsabilidad social empresarial: un compromiso necesario. Centro de Investigación de la Universidad del Pacífico.

Bobadilla, B. y Sandoval, S. (2015). La responsabilidad social y su influencia en la imagen institucional de la empresa de transporte Rodrigo Carranza S.A.C. Trujillo 2014 [Tesis de licenciatura, Universidad Privada Antenor Orrego] Repositorio institucional https://repositorio.upao.edu.pe/handle/20.50 $\underline{0.12759 / 1950}$

Castillo, C. (2005). La responsabilidad social de la empresa y los consumidores [Sesión de conferencia]. Encuentro Nacional de Organizaciones de Consumidores, Buenos Aires, Argentina.

Centro de la Investigación sobre la epidemiología de los desastres [CRED] (2017). Provides valuable information on the 41 impact for disasters. PAHO/WHO Emergencies News. https://www3.paho.org/disasters/newsletter/i ndex.php?option=com content\&amp;view=ar ticle\&amp;id=456:cred-ofrece-informacionestadistica-sobre-el-impacto-de-losdesastres\&amp; catid=214\&amp;|temid=285\&a mp;lang=en 
Comisión Nacional de Prevención de Riesgos y Atención de Emergencias. (2014). Gestión municipal del riesgo de desastres: normas y elementos básicos para su inclusión en el ordenamiento territorial. Énfasis en prevención, control y regulación territorial. Costa Rica.

George, D. y Mallery, P. (2003). SPSS for Windows step by step: a simple guide and reference (4.a ed.). 11.0 Update.

Hernández-Sampieri, R. y Mendoza, C. (2018). Metodología de la investigación. Editorial McGraw-Hill Educación.

Instituto Nacional de Geofísica del Perú [IGP] (2020). Instituto Geofísico del Perú instaló sistema para monitorear posibles huaicos en el verano. Gob.pe.

https://www.igp.gob.pe/versionanterior/instituto-geofisico-peru-instalosistema-monitorear-posibles-huaicos-verano$\underline{2020}$

Lavell, A. (2007). Apuntes para una reflexión institucional en países de la subregión andina sobre el enfoque de la gestión del riesgo. PREDECAN.

Ley 29664 (2011). Ley que crea el Sistema Nacional de Gestión de Desastres - SINAGERD. 08 de febrero de 2011.

Montañez, J. (2017). Responsabilidad social y el desarrollo sostenible en la Municipalidad Provincial de Yungay - 2017 [Tesis de maestría, Universidad César Vallejo] Repositorio institucional. https://repositorio.ucv.edu.pe/handle/20.500. $\underline{12692 / 12009}$

Ochante, P. (2018). Ejecución de obras públicas y responsabilidad social en la Municipalidad Provincial de Ica, 2018 [Tesis de Maestría, Universidad César Vallejo] Repositorio institucional.

https://repositorio.ucv.edu.pe/handle/20.500. $\underline{12692 / 29419}$

Oficina para la Coordinación de Asuntos Humanitarios de las Naciones Unidas [OCHA] (2020, 18 marzo). Desastres Naturales en América Latina y el Caribe, 2000/2019. World Relief Web.

https://reliefweb.int/report/world/desastresnaturales-en-am-rica-latina-y-el-caribe-2000$\underline{2019}$

Quispe, S. (2017). Responsabilidad social y gestión del riesgo de desastres de los empleados en la Municipalidad Provincial de Ica, Ica-2017 [Tesis de maestría. Universidad César Vallejo] Repositorio institucional.

https://repositorio.ucv.edu.pe/handle/20.500. $\underline{12692 / 9997}$

Rodríguez, J. (2006). Responsabilidad social corporativa: ¿Filantropía estratégica u otro modelo de empresa? Revista AECA, (74), 11-15.

Strand, H., Gates, S., Hegre, H. y Jones, M. (2003). Armed Conflict Dataset Codebook. PRIO 